\title{
THE EFFECT OF THE HEART'S POSITION ON THE ELECTROCARDIOGRAPHIC APPEARANCE OF VENTRICULAR EXTRASYSTOLES ${ }^{1}$
}

\author{
BY LOUIS N. KATZ AND WALTER ACKERMAN \\ (From the Cardiovascular Laboratory of the Department of Physiology, Michael Reese \\ Hospital, Chicago)
}

(Received for publication June 14, 1932)

Within recent years the "classical" interpretation of electrocardiograms of bundle branch block, which was based on Lewis's work on dogs (1), has been questioned. Wilson and his coworkers (2) came to the conclusion that the bundle branch actually involved was just the reverse of the "classical" interpretation. These conclusions were based on the relative time of initial negativity of the two ventricles in man in bundle branch block as presumably given by so-called "semi-direct" leads from the chest wall over the right and left ventricles. The criterion used in such measurements, namely the point in the QRS group of the electrocardiogram where the deflection first begins to rise, has not been shown conclusively to indicate the activation of the region beneath the electrode. The second line of evidence is based on the appearance in ordinary indirect leads of extrasystoles produced experimentally in a clinical case of pericardial fistulae and pericarditis (Barker, Macleod and Alexander (3)) recently confirmed on another subject by Marvin and Oughterson (4). The direction of the QRS group of the extrasystoles from the two ventricles was just the reverse of that anticipated on the classical theory of Lewis. The evidence, however, is not convincing that these hearts were normal in size and position.

Some years ago Fahr (5) claimed to have shown on theoretical grounds that the classical interpretation of bundle branch block reversed the bundle branches. It has furthermore been known for a long time that while right bundle branch block was the more common electrocardiographic diagnosis, yet at autopsy left bundle branch block was more common (cf. Taussig (6)). It must be pointed out that the electrocardiographic and autopsy findings in individual cases do not always agree -in some cases they do, (Hill (8), Taussig (6)), in others they do not, (Oppenheimer and Pardee (7), Mahain (14)). The problem is further complicated by the fact that the electrocardiographic evidence of bundle branch block can be present without any demonstrable anatomical lesion.

${ }^{1}$ Aided by the Emil and Fanny Wedeles Fund of the Michael Reese Hospital for the Study of Diseases of the Heart and Circulation. 
The foregoing argument concerning bundle branch block would apply with equal weight to extrasystoles of ventricular origin.

Recently, in reviewing the subject of bundle branch block one of us, (Katz (9)), came to the conclusion that some of the difficulty involved in interpreting the electrocardiogram in bundle branch block was due to variations in the position of the heart in the chest and in the amount and distribution of ventricular hypertrophy and dilatation. Evidence has been presented on the isolated preparation, (Boden and Neukirch (10)), in the open-chested dog, (Meek and Wilson (11)), and in man, (Nathanson (12)), that shifting the heart's position altered the direction and amplitude of the major initial deflection in the electrocardiogram. The changes were for the most part those anticipated, except when the heart was rotated on its own long axis.

In the present study an attempt was made to analyze the changes, produced in different positions of the heart, in the electrocardiographic appearance of ventricular extrasystoles, derived from fixed points in the dog's ventricle.

\section{EXPERIMENTAL METHODS}

The experiments were made on seven dogs, anesthetized with barbital, with open chest and artificial respiration. Pairs of platinum electrodes were inserted in four regions of the heart, to wit, the base of the right ventricle, the apex of the right ventricle, the base of the left ventricle and the apex of the left ventricle. The wires from these electrodes were coiled and suspended from an insulated rod. By means of a distributor the current for producing the extrasystoles was sent at will to any of these regions. A modified Lewis rotating current interrupter was employed to induce the extrasystoles. The rate of these stimuli was adjusted so that an extrasystole was induced every third or fifth beat at approximately the same point in diastole. In three of the experiments the heart rate was controlled by another set of commutators connected to the same rotating pole that had the commutators for the extrasystoles. In this fashion a constant exact placement of the extrasystole in the heart cycle was obtained. It was found, however, that this refinement was not necessary if the extrasystoles were placed late in diastole.

The position of the heart was adjusted by proper traction on three threads sewn into the heart, one in the apex, one on the lateral wall of the left ventricle, and one on the lateral wall of the right ventricle. Six positions of the heart were used, viz.:

1. Heart horizontal and apex pointing caudad.2

2. Heart's apex up to form an angle of approximately $50^{\circ}$ with the long axis of the body, apex pointing caudad.

3. Heart's apex up $25^{\circ}$, and to the left $30^{\circ}$ of the long axis of the body.

4. Heart's apex up $25^{\circ}$, and to the right $30^{\circ}$ of the long axis of the body.

5. Heart's apex up $25^{\circ}$, to the left $15^{\circ}$ of the long axis of the body, and heart rotated on its own long axis to bring right ventricle more anteriorly.

6. Heart's apex up $25^{\circ}$, to the right $15^{\circ}$ of the long axis of the body, and heart rotated on its own long axis to bring left ventricle more anteriorly.

While it was attempted to make the shifts as simple as possible, displace-

2 The normal position of the heart's apex is $25^{\circ}$ up from the horizontal. 
ments in other planes and rotation in moving the heart between positions in the first two pairs of positions ( 1 and 2, 3 and 4) could not be avoided.

The electrocardiograms, usual leads, were taken in each of these positions in quick succession in the following order: Lead I was taken with the distributor arranged to give extrasystoles first in the base of the right ventricle, then in the apex of the right ventricle, then in the base of the left ventricle, and then in the apex of the left ventricle. Leads II and III were taken with extrasystoles produced in the same order. Each record was standardized.

The amplitude of the major initial deflection in each record was measured and corrected for standardization. When a $P$ wave fused with the $Q R S$ group, this was taken into account, to avoid error with large $P$ waves. A positive major initial deflection was designated by plus, a negative one by minus. When the two phases were of almost equal amplitude they were both measured and placed with proper sign one after the other. These measurements are assembled in Table I. The change in the so-called electrical axis associated with the shift in the heart's position was determined by using Einthoven's vector analysis. The shift in electrical axis was determined between each pair of heart's positions, viz., positions 1 and 2 , positions 3 and 4 , and positions 5 and 6 above. This was facilitated by means of the twelve diagrams in Figure 1. Since it has been shown by Zeisler (13) that Einthoven's vector is only a rough estimate, and since the three leads were not taken simultaneously nor are the peaks of the major deflections in the three leads necessarily homologous points, this analysis is to be considered no more than a rough estimate of the changes in the manifest potential. Nevertheless, as will be shown below, striking changes were found.

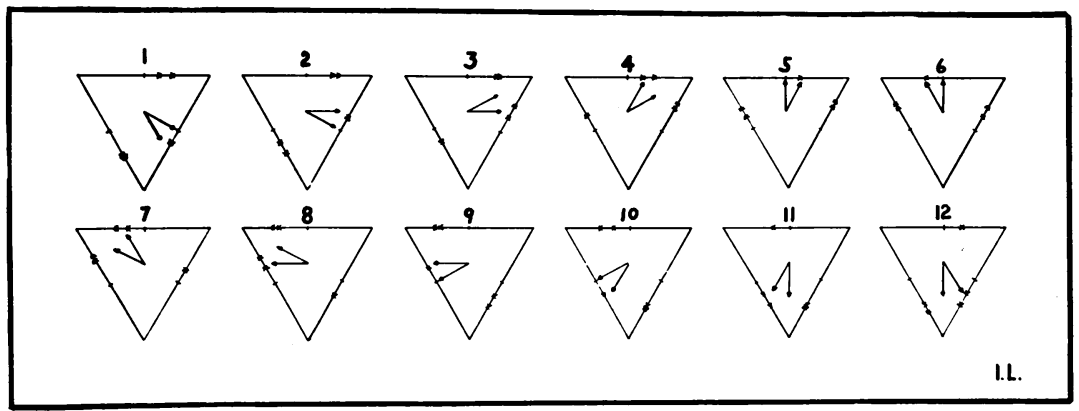

Fig. 1. Diagram of Vector Analysis of the "Manifest Potential" on Einthoven's Triangle, with the "Manifest Potential" Shifted Around $360^{\circ}$ in Twelve Stages.

The arrows on the three sides of the triangle give the direction and relative magnitude of the projection of the "manifest potential" in each lead. As pointed out in the text, Einthoven's analysis is only a rough estimate.

\section{RESULTS}

It was found that as the apex of the heart was raised $50^{\circ}$ from the horizontal, the amplitude of the major initial deflection of the sinus and extrasystolic beats decreased (cf. Table I and the complete protocol of Experiment 8 reassembled in Figures 2, 3, 4 and 5 to show the variation in configuration of the sinus beats and of the same extrasystoles in the 


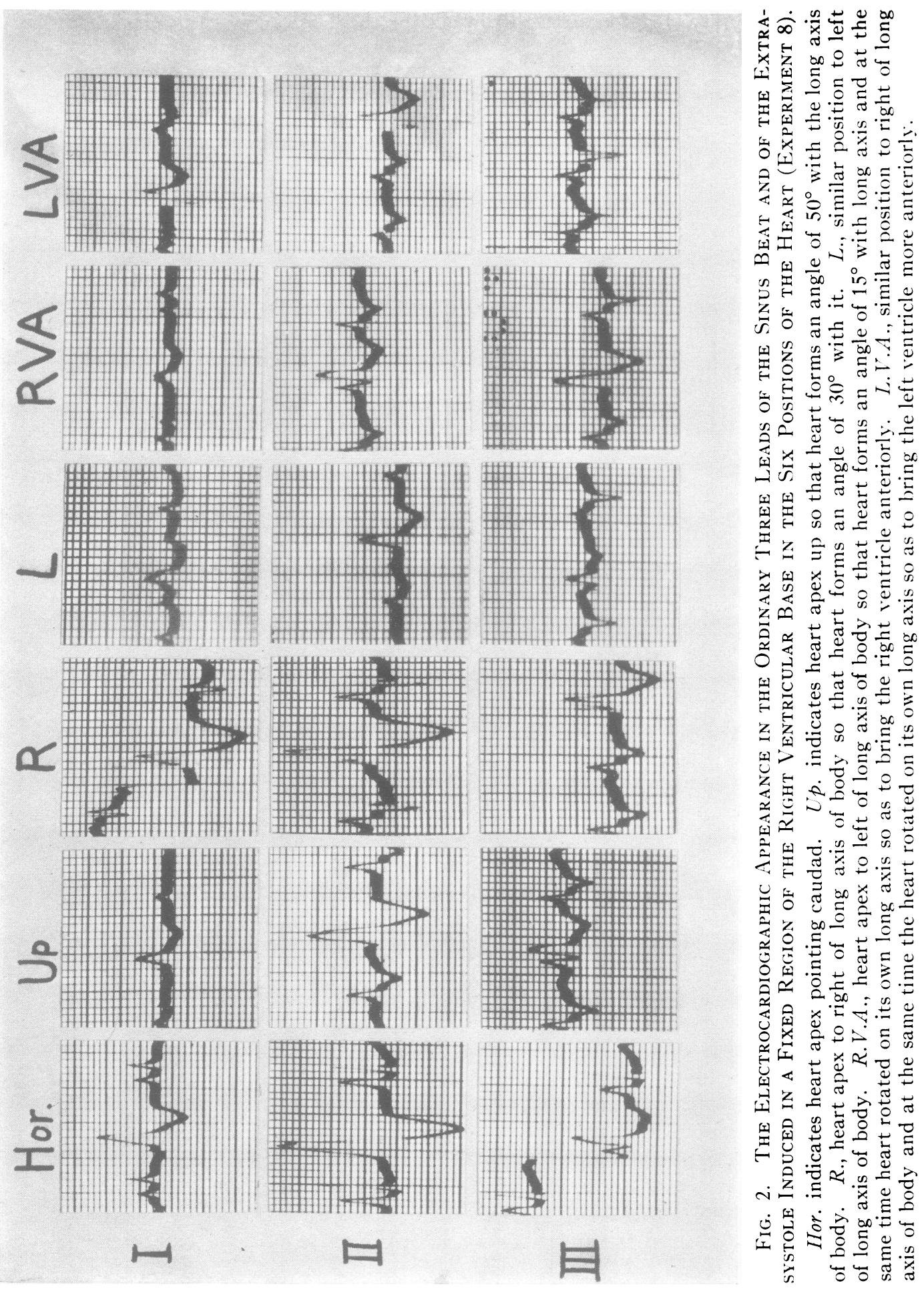




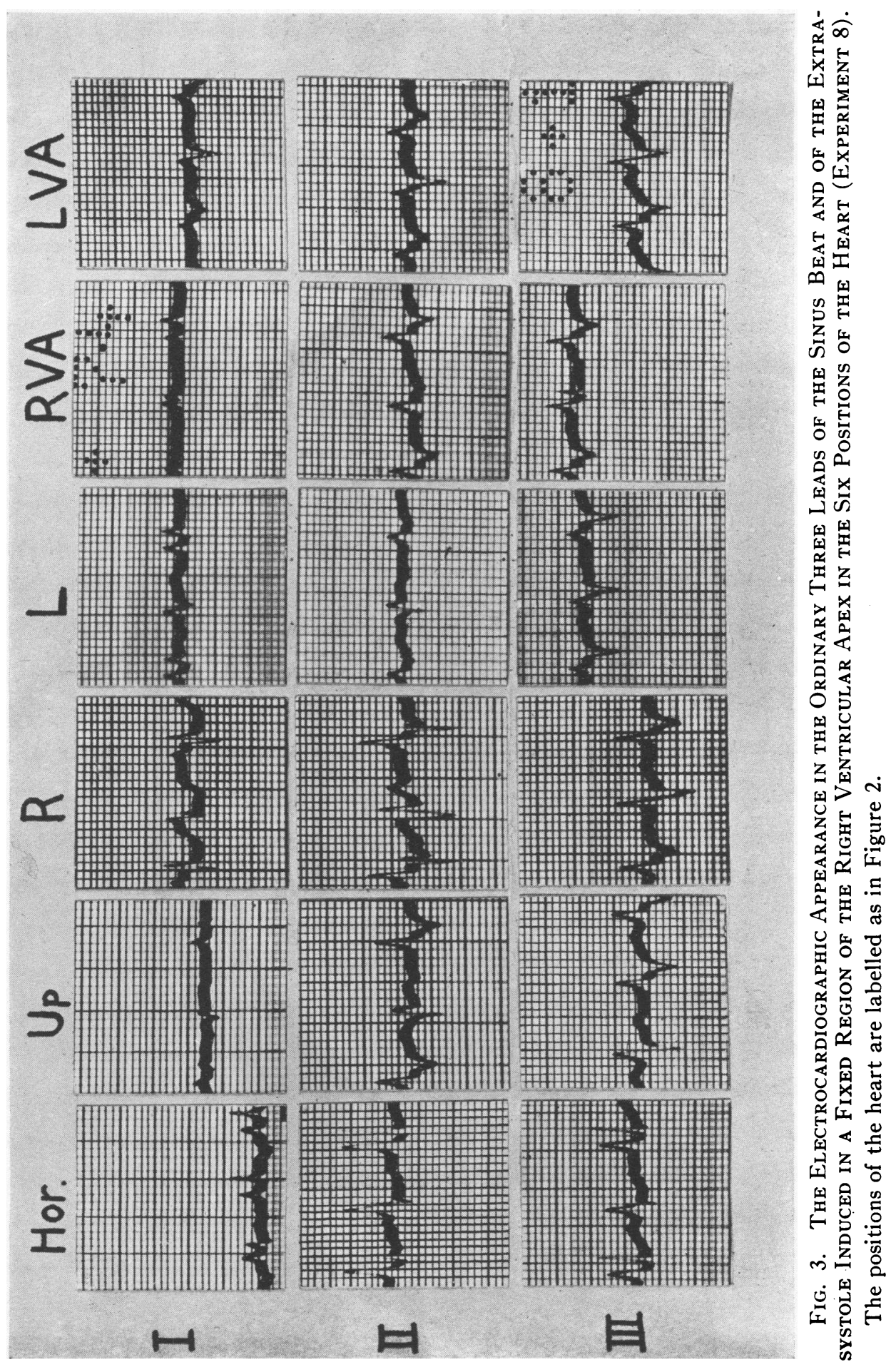




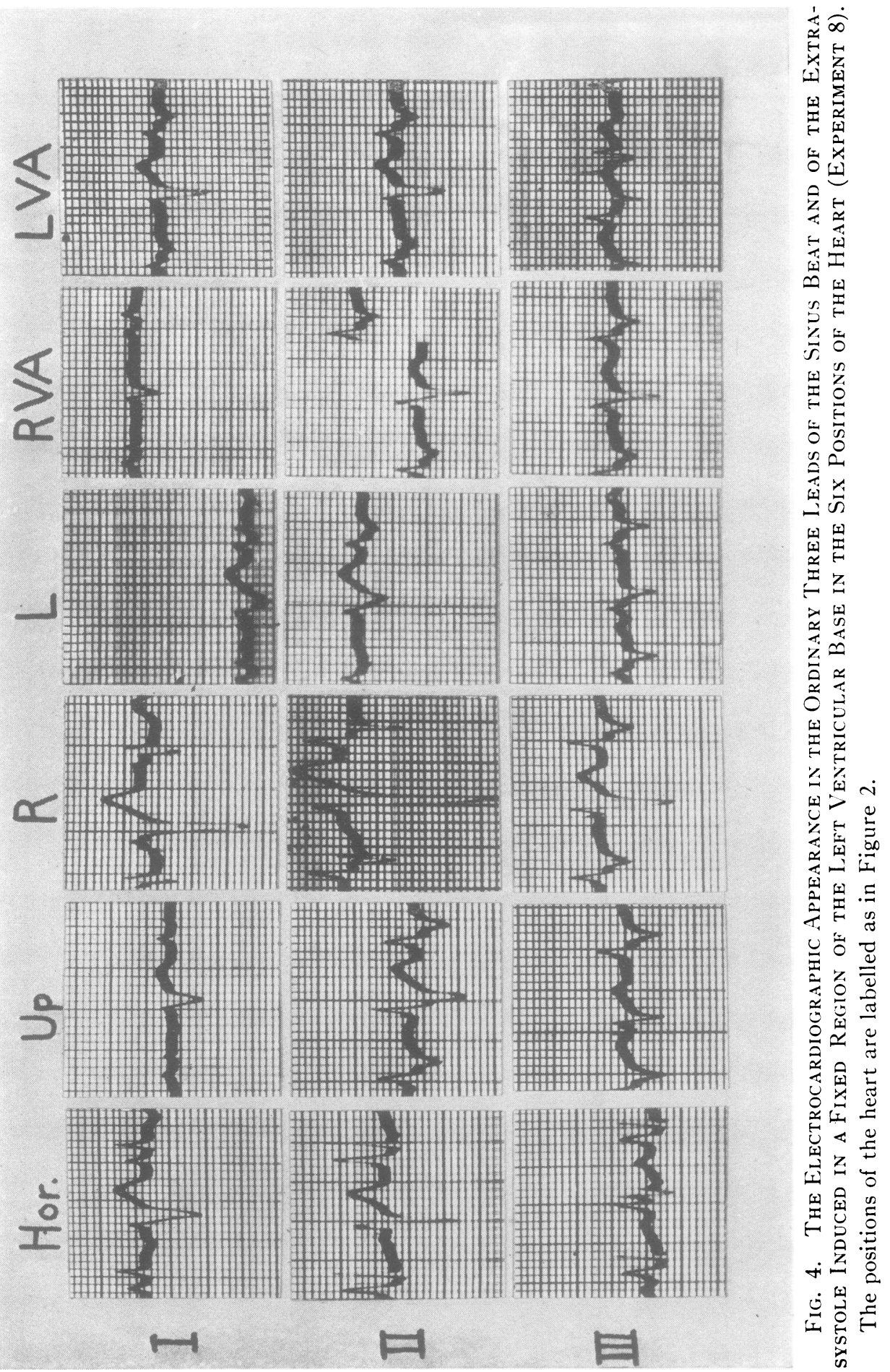




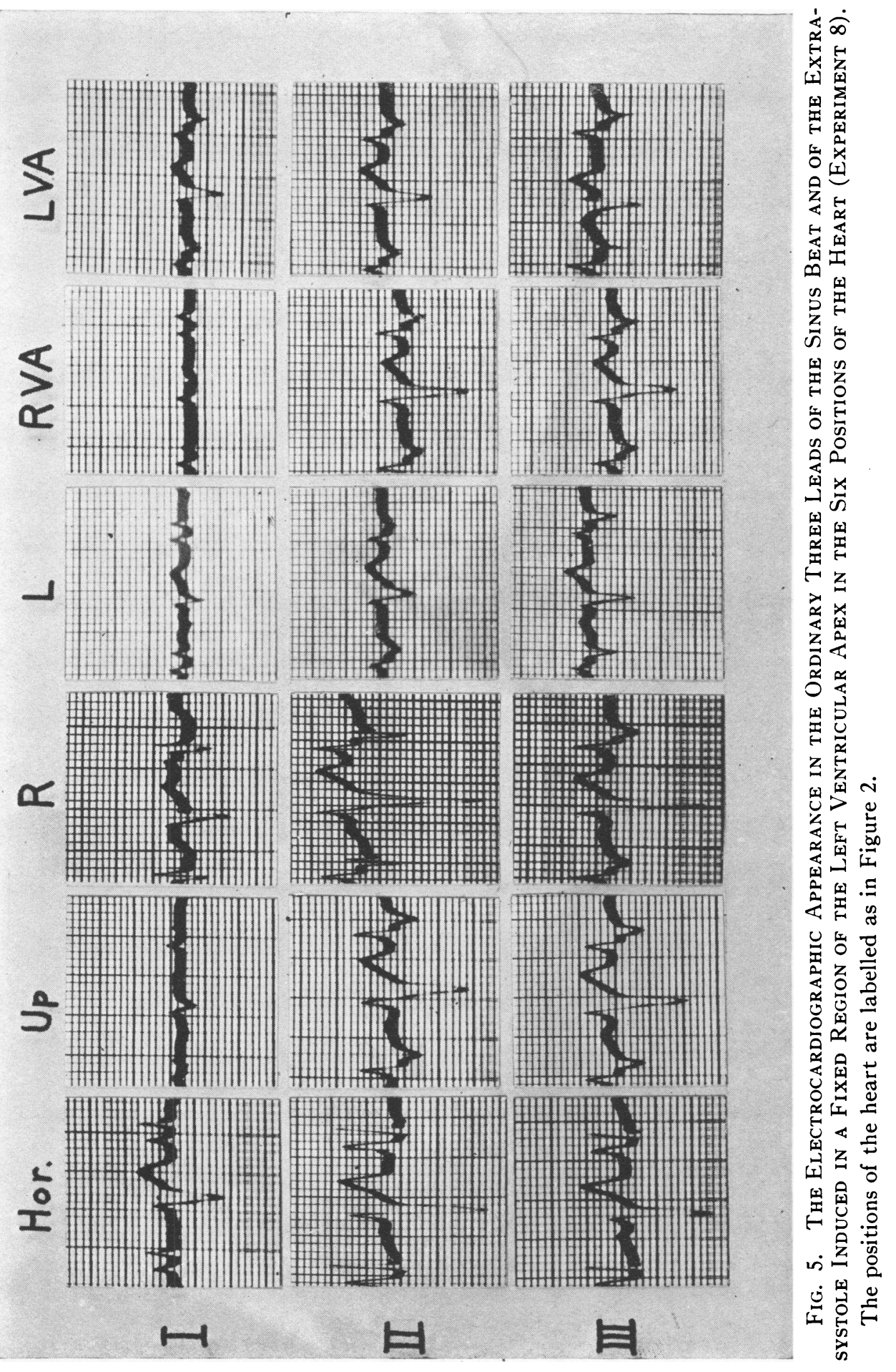


TABLE I

Changes in the amplitude of the major initial deflection of sinus and extrasystolic beats with shifts in the heart's position*

\begin{tabular}{|c|c|c|c|c|c|c|c|}
\hline $\begin{array}{c}\text { Experi- } \\
\text { ment } \\
\text { number }\end{array}$ & Lead & $\begin{array}{c}\text { Heart } \\
\text { hori- } \\
\text { zontal }\end{array}$ & $\begin{array}{l}\text { Heart } \\
\text { apex 50० } \\
\text { up from } \\
\text { long axis } \\
\text { of body }\end{array}$ & $\begin{array}{l}\text { Heart apex } \\
30^{\circ} \text { left } \\
\text { of the } \\
\text { long axis } \\
\text { of body }\end{array}$ & $\begin{array}{l}\text { Heart apex } \\
30^{\circ} \text { right } \\
\text { of the } \\
\text { long axis } \\
\text { of body }\end{array}$ & $\begin{array}{l}\text { Heart apex 15० } \\
\text { left of long } \\
\text { axis of body } \\
\text { and heart ro- } \\
\text { tated on its } \\
\text { own long axis } \\
\text { to bring right } \\
\text { ventricle more } \\
\text { anteriorly }\end{array}$ & $\begin{array}{l}\text { Heart apex } 15^{\circ} \\
\text { right of long } \\
\text { axis of body } \\
\text { and heart ro- } \\
\text { tated on its } \\
\text { own long axis } \\
\text { to bring left } \\
\text { ventricle more } \\
\text { anteriorly }\end{array}$ \\
\hline $1 \mathrm{~N}$ & $\begin{array}{r}\text { I } \\
\text { II } \\
\text { III }\end{array}$ & $\begin{array}{l}+1.5 \\
+13 \\
+12\end{array}$ & $\begin{array}{l}+1 \\
-1+1 \\
-1\end{array}$ & $\begin{array}{l}+6.5 \\
+4 \\
-3\end{array}$ & $\begin{array}{l}-1 \\
+17 \\
+15.5\end{array}$ & $\begin{array}{l}-1 \\
+2.5 \\
+13.5\end{array}$ & $\begin{array}{l}+2.5 \\
+6.5 \\
+6\end{array}$ \\
\hline $1 \mathrm{RB}$ & $\begin{array}{r}\text { I } \\
\text { II } \\
\text { II I } \\
\end{array}$ & +6 & $\begin{array}{l}+3.5 \\
+6 \\
-2 \\
\end{array}$ & $\begin{array}{l}+13 \\
+6 \\
-8 \\
\end{array}$ & $\begin{array}{l}+3.5 \\
+15 \\
+11\end{array}$ & $\begin{array}{l}+4 \\
+5.5 \\
+5.5\end{array}$ & $\begin{array}{l}+4.5 \\
+6 \\
+3\end{array}$ \\
\hline $1 \mathrm{RA}$ & $\begin{array}{r}\text { I } \\
\text { II } \\
\text { III }\end{array}$ & $\begin{array}{r}+6 \\
+4 \\
\end{array}$ & $\begin{array}{l}+2.5 \\
+2 \\
-1 \\
\end{array}$ & $\begin{array}{r}+13 \\
+6 \\
-8 \\
\end{array}$ & $\begin{array}{l}+3 \\
+17 \\
+11 \\
\end{array}$ & $\begin{array}{l}+3 \\
+3.5 \\
+4 \\
\end{array}$ & $\begin{array}{l}+4 \\
+6 \\
-3.5 \\
\end{array}$ \\
\hline $1 \mathrm{LB}$ & $\begin{array}{r}\text { I } \\
\text { II } \\
\text { III } \\
\end{array}$ & $\begin{array}{l}-3.5 \\
+10 \\
\end{array}$ & $\begin{array}{l}-3 \\
+3 \\
+5.5 \\
\end{array}$ & $\begin{array}{l}-13.5 \\
+4 \\
+10\end{array}$ & $\begin{array}{l}-7 \\
+4-5 \\
+9\end{array}$ & $\begin{array}{l}-5.5 \\
-2.5 \\
+9\end{array}$ & $\begin{array}{l}-1 \\
-12 \\
+14\end{array}$ \\
\hline $1 \mathrm{LA}$ & $\begin{array}{r}\text { I } \\
\text { II } \\
\text { III } \\
\end{array}$ & $\begin{array}{l}-3.5 \\
-2+3.5 \\
+6\end{array}$ & $\begin{array}{l}-1 \\
-2 \\
-1\end{array}$ & $\begin{array}{l}-3 \\
-4 \\
+3 \\
\end{array}$ & $\begin{array}{l}+7 \\
+10 \\
+13\end{array}$ & $\begin{array}{l}-2 \\
+2 \\
+11\end{array}$ & $\begin{array}{l}-1 \\
-1 \\
+3.5\end{array}$ \\
\hline $3 \mathrm{~N}$ & $\begin{array}{r}\text { I } \\
\text { II } \\
\text { III } \\
\end{array}$ & $\begin{array}{l}-5+6 \\
+17 \\
+19 \\
\end{array}$ & $\begin{array}{l}+5 \\
+4 \\
+1.5 \\
\end{array}$ & $\begin{array}{l}+2 \\
+18 \\
+7.5 \\
\end{array}$ & $\begin{array}{l}-8.5+7 \\
+24 \\
+13 \\
\end{array}$ & & \\
\hline $3 \mathrm{RH}$ & $\begin{array}{r}\text { I } \\
\text { II } \\
\text { III } \\
\end{array}$ & $\begin{array}{r}+6 \\
+28 \\
+21 \\
\end{array}$ & +4 & $\begin{array}{l}+4.5 \\
+18.5 \\
+\quad 7.5 \\
\end{array}$ & $\begin{array}{l}+9 \\
+28.5 \\
+18.5 \\
\end{array}$ & & \\
\hline $3 \mathrm{RA}$ & $\begin{array}{r}\text { I } \\
\text { II } \\
\text { III } \\
\end{array}$ & & & $\begin{array}{l}+2 \\
-9\end{array}$ & $\begin{array}{l}-5 \\
-13 \\
-13 \\
\end{array}$ & & \\
\hline $3 \mathrm{LB}$ & $\begin{array}{r}\text { I } \\
\text { II } \\
\text { III } \\
\end{array}$ & & & $\begin{array}{l}-3 \\
-10 \\
-4 \\
\end{array}$ & $\begin{array}{l}-10 \\
-28.5 \\
-13 \\
\end{array}$ & & \\
\hline $3 \mathrm{LA}$ & $\begin{array}{r}\text { I } \\
\text { II } \\
\text { II I }\end{array}$ & & & $\begin{array}{l}-3 \\
-8 \\
-7.5 \\
\end{array}$ & $\begin{array}{l}-11.5 \\
-24 \\
-14 \\
\end{array}$ & & \\
\hline
\end{tabular}

* $\mathrm{N}=$ Normal complex.

$\mathrm{RB}=$ Extrasystole from right base of heart.

$\mathrm{RA}=$ Extrasystole from right apex of heart.

$\mathrm{LB}=$ Extrasystole from left base of heart.

$\mathrm{L}$ A $=$ Extrasystole from left apex of heart.

+ indicates an upright deflection.

- indicates an inverted deflection.

When a diphasic QRS is present the amplitudes of both phases are given, in order of their appearance. 
TABLE I (continued)

\begin{tabular}{|c|c|c|c|c|c|c|c|}
\hline $\begin{array}{c}\text { Experi- } \\
\text { ment } \\
\text { number }\end{array}$ & Lead & $\begin{array}{c}\text { Heart } \\
\text { hori- } \\
\text { zontal }\end{array}$ & $\begin{array}{l}\text { Heart } \\
\text { apex 500 } \\
\text { up from } \\
\text { long axis } \\
\text { of body }\end{array}$ & $\begin{array}{c}\text { Heart apex } \\
30^{\circ} \text { left } \\
\text { of the } \\
\text { long axis } \\
\text { of body }\end{array}$ & $\begin{array}{c}\text { Heart apex } \\
30^{\circ} \text { right } \\
\text { of the } \\
\text { long axis } \\
\text { of body }\end{array}$ & $\begin{array}{l}\text { Heart apex 150 } \\
\text { left of long } \\
\text { axis of body } \\
\text { and heart ro- } \\
\text { tated on its } \\
\text { own long axis } \\
\text { to bring right } \\
\text { ventricle more } \\
\text { anteriorly }\end{array}$ & \begin{tabular}{|} 
Heart apex 15० \\
right of long \\
axis of body \\
and heart ro- \\
tated on its \\
own long axis \\
to bring left \\
ventricle more \\
anteriorly
\end{tabular} \\
\hline $4 \mathrm{~N}$ & $\begin{array}{r}\text { I } \\
\text { II } \\
\text { III }\end{array}$ & $\begin{array}{l}+8 \\
+8.5 \\
-4\end{array}$ & $\begin{array}{l}-2+2 \\
-2 \\
-4.5\end{array}$ & $\begin{array}{l}+13 \\
+7.5 \\
-8\end{array}$ & $\begin{array}{l}-2+2 \\
+6 \\
+6\end{array}$ & $\begin{array}{l}+17.5 \\
+4 \\
-12.5\end{array}$ & $\begin{array}{l}-3+2 \\
-5 \\
-3.5\end{array}$ \\
\hline $4 \mathrm{RB}$ & \begin{tabular}{|r|} 
I \\
II \\
III
\end{tabular} & $\begin{array}{l}+6.5 \\
+7 \\
-3\end{array}$ & $\begin{array}{l}+2 \\
+2 \\
+2.5\end{array}$ & $\begin{array}{l}+16 \\
+14 \\
-10\end{array}$ & $\begin{array}{l}+5 \\
+11 \\
+5\end{array}$ & & \\
\hline $4 \mathrm{LB}$ & \begin{tabular}{|r|} 
I \\
II \\
III
\end{tabular} & $\begin{array}{l}+5 \\
+2 \\
+5.5\end{array}$ & $\begin{array}{l}+4 \\
+4 \\
+5\end{array}$ & & & & \\
\hline $4 \mathrm{LA}$ & \begin{tabular}{r|} 
I \\
II \\
III
\end{tabular} & $\begin{array}{l}-2 \\
-8.5 \\
-4\end{array}$ & $\begin{array}{l}-0.5 \\
-4 \\
-9\end{array}$ & & & & \\
\hline $6 \mathrm{~N}$ & $\begin{array}{r}\text { I } \\
\text { II } \\
\text { III }\end{array}$ & $\begin{array}{l}+1 \\
+9 \\
+6.5\end{array}$ & $\begin{array}{l}+0.5 \\
+1 \\
-2.5\end{array}$ & $\begin{array}{l}+1 \\
+0.5 \\
-3\end{array}$ & $\begin{array}{l}-0.5 \\
+0.5 \\
+4.0\end{array}$ & $\begin{array}{l}+0.5 \\
+2.5 \\
+4.5\end{array}$ & $\begin{array}{l}+1 \\
-3 \\
-4\end{array}$ \\
\hline $6 \mathrm{RB}$ & $\begin{array}{r}\text { I } \\
\text { II } \\
\text { III }\end{array}$ & $\begin{array}{l}+3.5 \\
+4 \\
-4\end{array}$ & $\begin{array}{l}+2 \\
+1 \\
-5\end{array}$ & $\begin{array}{l}+2 \\
+1 \\
-4\end{array}$ & $\begin{array}{l}+4.5 \\
+6 \\
+5\end{array}$ & $\begin{array}{l}+2 \\
+4 \\
+9\end{array}$ & $\begin{array}{l}+1 \\
-12 \\
-14\end{array}$ \\
\hline $6 \mathrm{RA}$ & $\begin{array}{r}\text { I } \\
\text { II } \\
\text { III } \\
\end{array}$ & $\begin{array}{l}+4 \\
+5 \\
-7\end{array}$ & $\begin{array}{l}+2 \\
+1 \\
-3\end{array}$ & $\begin{array}{l}+2 \\
+1 \\
-3 \\
\end{array}$ & $\begin{array}{l}+3 \\
+5 \\
-5\end{array}$ & $\begin{array}{l}-1 \\
+3.5 \\
+10.5\end{array}$ & $\begin{array}{l}+2.5 \\
-8 \\
-10\end{array}$ \\
\hline $6 \mathrm{LB}$ & $\begin{array}{r}\text { I } \\
\text { II } \\
\text { III }\end{array}$ & $\begin{array}{l}-4 \\
+8.5 \\
+11\end{array}$ & $\begin{array}{l}-2 \\
+2.5 \\
+5.5\end{array}$ & $\begin{array}{l}-2 \\
+1 \\
+2\end{array}$ & $\begin{array}{l}-4.5 \\
-4.5 \\
+8\end{array}$ & $\begin{array}{l}-3.5 \\
-2.5 \\
+10\end{array}$ & $\begin{array}{l}-2 \\
+5 \\
+7\end{array}$ \\
\hline $6 \mathrm{LA}$ & $\begin{array}{r}\text { I } \\
\text { II } \\
\text { III }\end{array}$ & $\begin{array}{l}+1 \\
-16.5 \\
-15\end{array}$ & $\begin{array}{l}+0.5 \\
-3 \\
-6.5\end{array}$ & $\begin{array}{l}-0.5 \\
-4 \\
-8\end{array}$ & $\begin{array}{l}-1 \\
-6 \\
-16\end{array}$ & $\begin{array}{l}+2.5 \\
-2 \\
-12\end{array}$ & $\begin{array}{l}-2.5 \\
-8 \\
-9.5\end{array}$ \\
\hline $7 \mathrm{~N}$ & $\begin{array}{r}\text { I } \\
\text { II } \\
\text { III } \\
\end{array}$ & $\begin{array}{r}+2 \\
+9 \\
+14 \\
\end{array}$ & $\begin{array}{l}+1.5 \\
+7 \\
+8\end{array}$ & $\begin{array}{l}+3 \\
+8 \\
+5 \\
\end{array}$ & $\begin{array}{l}+2 \\
+14 \\
+13\end{array}$ & $\begin{array}{l}+8.5 \\
+9 \\
+6 \\
\end{array}$ & $\begin{array}{l}+3.5 \\
+13 \\
+4 \\
\end{array}$ \\
\hline $7 \mathrm{RB}$ & $\begin{array}{r}\text { I } \\
\text { II } \\
\text { II I } \\
\end{array}$ & $\begin{array}{r}+3.5 \\
+6.5 \\
+\quad 8.5 \\
\end{array}$ & $\begin{array}{l}+2.5 \\
+6.5 \\
+6.5\end{array}$ & $\begin{array}{r}+4.5 \\
+7.5 \\
+4.5 \\
\end{array}$ & $\begin{array}{l}+10 \\
+26 \\
+14\end{array}$ & $\begin{array}{l}+4.5 \\
+14 \\
+12\end{array}$ & $\begin{array}{l}+8 \\
+11 \\
-5 \\
\end{array}$ \\
\hline $7 \mathrm{RA}$ & \begin{tabular}{r|} 
I \\
II \\
III \\
\end{tabular} & $\begin{array}{l}+4 \\
+5 \\
-6\end{array}$ & $\begin{array}{l}+2.5 \\
+3.5 \\
-1.5\end{array}$ & $\begin{array}{l}+5.5 \\
+3 \\
-6.5\end{array}$ & $\begin{array}{l}+10 \\
+13 \\
+4\end{array}$ & $\begin{array}{l}+8 \\
+10 \\
+3\end{array}$ & $\begin{array}{l}+8 \\
-6.5 \\
-13 \\
\end{array}$ \\
\hline $7 \mathrm{LB}$ & $\begin{array}{r}\text { I } \\
\text { II } \\
\text { III }\end{array}$ & $\begin{array}{l}-3 \\
-6.5 \\
-3\end{array}$ & $\begin{array}{l}-4 \\
-7 \\
-3\end{array}$ & $\begin{array}{l}-7 \\
-6 \\
+5\end{array}$ & $\begin{array}{l}-9 \\
-19 \\
-11\end{array}$ & $\begin{array}{l}-8.5 \\
-6 \\
+8.5\end{array}$ & $\begin{array}{l}-12 \\
-16 \\
+8.5\end{array}$ \\
\hline $7 \mathrm{LA}$ & $\begin{array}{r}\text { I } \\
\text { II } \\
\text { III }\end{array}$ & $\begin{array}{l}-1 \\
-4.5 \\
-8\end{array}$ & $\begin{array}{l}-0.5 \\
-7 \\
-6\end{array}$ & $\begin{array}{l}-1.5 \\
-7.5 \\
-4\end{array}$ & $\begin{array}{l}-5 \\
-19 \\
-13\end{array}$ & $\begin{array}{l}-5.5 \\
-11.5 \\
-8\end{array}$ & $\begin{array}{l}-6.5 \\
-13 \\
-8\end{array}$ \\
\hline
\end{tabular}


TABLE I (continued)

\begin{tabular}{|c|c|c|c|c|c|c|c|}
\hline $\begin{array}{c}\text { Experi- } \\
\text { ment } \\
\text { number }\end{array}$ & Lead & $\begin{array}{l}\text { Heart } \\
\text { hori- } \\
\text { zontal }\end{array}$ & $\begin{array}{l}\text { Heart } \\
\text { apex 50 } \\
\text { up from } \\
\text { long axis } \\
\text { of body }\end{array}$ & $\begin{array}{l}\text { Heart apex } \\
50^{\circ} \text { left } \\
\text { of the } \\
\text { long axis } \\
\text { of body }\end{array}$ & $\begin{array}{l}\text { Heart apex } \\
30^{\circ} \text { right } \\
\text { of the } \\
\text { long axis } \\
\text { of body }\end{array}$ & $\begin{array}{l}\text { Ileart apex } 15^{\circ} \\
\text { left of long } \\
\text { axis of body } \\
\text { and heart ro- } \\
\text { tated on its } \\
\text { own long axis } \\
\text { to bring right } \\
\text { ventricle more } \\
\text { anteriorly }\end{array}$ & $\begin{array}{l}\text { Heart apex } 15^{\circ} \\
\text { right of long } \\
\text { axis of body } \\
\text { and heart ro- } \\
\text { tated on its } \\
\text { own long axis } \\
\text { to bring left } \\
\text { ventricle more } \\
\text { anteriorly } \\
\end{array}$ \\
\hline $8 \mathrm{~N}$ & \begin{tabular}{r|} 
I \\
II \\
III \\
\end{tabular} & $\begin{array}{l}+2 \\
+6 \\
+4 \\
\end{array}$ & $\begin{array}{l}+0.5 \\
-2.5 \\
-2\end{array}$ & $\begin{array}{l}+0.5 \\
-2 \\
-4\end{array}$ & $\begin{array}{l}-2.5+3.5 \\
-5 \\
-1.5\end{array}$ & $\begin{array}{l}+1 \\
-2 \\
-2\end{array}$ & $\begin{array}{l}-1 \\
-1 \\
-3\end{array}$ \\
\hline $8 \mathrm{RB}$ & \begin{tabular}{r|} 
I \\
II \\
III
\end{tabular} & $\begin{array}{l}+4 \\
+12 \\
+6\end{array}$ & $\begin{array}{l}+2.5 \\
+9 \\
+3.5\end{array}$ & $\begin{array}{l}+3 \\
+5 \\
+\quad 2\end{array}$ & $\begin{array}{l}+8 \\
+9 \\
+7\end{array}$ & $\begin{array}{l}+1.5 \\
+7 \\
+7\end{array}$ & $\begin{array}{l}+3 \\
+4 \\
-4\end{array}$ \\
\hline $8 \mathrm{RA}$ & $\begin{array}{r}\text { I } \\
\text { II } \\
\text { III }\end{array}$ & $\begin{array}{l}+1 \\
-1 \\
-1\end{array}$ & $\begin{array}{l}-0.5 \\
-4 \\
-3.5\end{array}$ & $\begin{array}{l}-1 \\
-2.5 \\
-3.5\end{array}$ & $\begin{array}{l}-2.5 \\
-7 \\
-3.5\end{array}$ & $\begin{array}{l}+1 \\
+3 \\
+\quad 2\end{array}$ & $\begin{array}{l}-3 \\
-5 \\
-3\end{array}$ \\
\hline $8 \mathrm{LB}$ & $\begin{array}{r}\text { I } \\
\text { II } \\
\text { III } \\
\end{array}$ & $\begin{array}{l}-3.5 \\
-6.5 \\
-2 \\
\end{array}$ & $\begin{array}{l}-2 \\
-4.5 \\
-3 \\
\end{array}$ & $\begin{array}{l}-4 \\
-3.5 \\
+0.5 \\
\end{array}$ & $\begin{array}{l}-11.5 \\
-22 \\
-10\end{array}$ & $\begin{array}{l}+17.5 \\
+4 \\
-12.5\end{array}$ & $\begin{array}{l}-7 \\
-6 \\
-2 \\
\end{array}$ \\
\hline $8 \mathrm{LA}$ & $\begin{array}{r}\text { I } \\
\text { II } \\
\text { II I } \\
\end{array}$ & $\begin{array}{l}-2.5 \\
-9 \\
-7\end{array}$ & $\begin{array}{l}-0.5 \\
-8 \\
-7\end{array}$ & $\begin{array}{l}-2 \\
-3.5 \\
-4.5\end{array}$ & $\begin{array}{l}-6 \\
-18 \\
-11\end{array}$ & $\begin{array}{l}+0.5 \\
-8 \\
-8\end{array}$ & $\begin{array}{l}-4 \\
-6 \\
-6\end{array}$ \\
\hline $9 \mathrm{~N}$ & $\begin{array}{r}\text { I } \\
\text { II } \\
\text { II I } \\
\end{array}$ & & & $\begin{array}{l}+7 \\
+7 \\
-3.5 \\
\end{array}$ & $\begin{array}{l}-2.5 \\
+7 \\
+7\end{array}$ & & \\
\hline $9 \mathrm{RA}$ & \begin{tabular}{r|} 
I \\
II \\
III
\end{tabular} & & & $\begin{array}{l}+10 \\
+11 \\
+2-2.5\end{array}$ & $\begin{array}{l}+12 \\
+16 \\
+9\end{array}$ & & \\
\hline
\end{tabular}

various positions of the heart). The variability in the shift of the electrical axis to the right or left is shown in Table II. The shifts of the electrical axes of the various extrasystoles in a single experiment were not proportional to each other nor to the shift in the electrical axis of the sinus beat. In fact, in practically every one of the experiments the shift in electrical axis was in an opposite direction in some beats to that in others of different origin.

Usually the deflections were larger when the apex of the heart was to the right of the long axis of the body than when the apex was to the left. The deflections were also larger when the right ventricle was rotated anteriorly than when the left ventricle was rotated anteriorly, although there were many exceptions (Table I).

It was found that moving the heart's apex from right to left in relation to the long axis of the body with or without rotation of the heart on its own long axis (viz., positions 3 and 4, 5 and 6) also gave a lack of proportionality in amount and a discordancy in direction of the shift of the electrical axis of the various types of beats (Table II). Furthermore, 
TABLE II

Shift in electrical axis

\begin{tabular}{|c|c|c|c|c|}
\hline $\begin{array}{c}\text { Experi- } \\
\text { ment } \\
\text { number }\end{array}$ & $\underset{\text { ism* }}{\text { Mechan- }}$ & $\begin{array}{l}\text { Shift of heart } \\
\text { from horizontal up }\end{array}$ & $\begin{array}{l}\text { Shift of heart } \\
\text { from right to left }\end{array}$ & $\begin{array}{l}\text { Shift of heart from right to left } \\
\text { plus rotation of heart on its own } \\
\text { long axis from left ventricle } \\
\text { anterior to right ventricle anterior }\end{array}$ \\
\hline 1 & $\begin{array}{l}\text { Normal } \\
\text { RB } \\
\text { RA } \\
\text { LB } \\
\text { LA }\end{array}$ & $\begin{array}{l}\text { Left } \\
\text { Left } \\
\text { Left } \\
\text { Right }\end{array}$ & $\begin{array}{l}\text { Left } \\
\text { Left } \\
\text { Left } \\
\text { Right } \\
\text { Right }\end{array}$ & $\begin{array}{l}\text { Right } \\
\text { Left } \\
\text { Right } \\
\text { Left } \\
\text { Right }\end{array}$ \\
\hline 3 & $\begin{array}{l}\text { Normal } \\
\text { RB } \\
\text { RA } \\
\text { LB } \\
\text { LA }\end{array}$ & Left & $\begin{array}{l}\text { Left } \\
\text { Left } \\
\text { Right } \\
\text { Left } \\
\text { None }\end{array}$ & \\
\hline 4 & $\begin{array}{l}\text { Normal } \\
\text { RB } \\
\text { LB } \\
\text { LA }\end{array}$ & $\begin{array}{l}\text { Left } \\
\text { Right } \\
\text { Right }\end{array}$ & $\begin{array}{l}\text { Left } \\
\text { Left }\end{array}$ & Right \\
\hline 6 & $\begin{array}{l}\text { Normal } \\
\text { RB } \\
\text { RA } \\
\text { LB } \\
\text { LA }\end{array}$ & $\begin{array}{l}\text { Left } \\
\text { None } \\
\text { Right } \\
\text { Left } \\
\text { Right }\end{array}$ & $\begin{array}{l}\text { Left } \\
\text { Left } \\
\text { None } \\
\text { Left } \\
\text { Left }\end{array}$ & $\begin{array}{l}\text { Right } \\
\text { Right } \\
\text { Right } \\
\text { Right } \\
\text { Right }\end{array}$ \\
\hline 7 & $\begin{array}{l}\text { Normal } \\
\text { RB } \\
\text { RA } \\
\text { LB } \\
\text { LA }\end{array}$ & $\begin{array}{l}\text { Left } \\
\text { None } \\
\text { Right } \\
\text { None } \\
\text { Left }\end{array}$ & $\begin{array}{l}\text { Left } \\
\text { Left } \\
\text { Left } \\
\text { Left }\end{array}$ & $\begin{array}{l}\text { Left } \\
\text { Right } \\
\text { Right } \\
\text { Left } \\
\text { None }\end{array}$ \\
\hline 8 & $\begin{array}{l}\text { Normal } \\
\text { RB } \\
\text { RA } \\
\text { LB } \\
\text { LA. }\end{array}$ & $\begin{array}{l}\text { Left } \\
\text { None } \\
\text { Left } \\
\text { Right } \\
\text { Right }\end{array}$ & $\begin{array}{l}\text { Right } \\
\text { None } \\
\text { Right } \\
\text { Left } \\
\text { Left }\end{array}$ & $\begin{array}{l}\text { Right } \\
\text { Right } \\
\text { Right }\left(180^{\circ}\right) \\
\text { Right } \\
\text { Right }\end{array}$ \\
\hline 9 & $\begin{array}{l}\text { Normal } \\
\text { RA }\end{array}$ & & $\begin{array}{l}\text { Left } \\
\text { Left }\end{array}$ & \\
\hline
\end{tabular}

* Normal equals sinus rhythm.

$\mathrm{RB}=$ Extrasystole from right base.

$\mathrm{RA}=$ Extrasystole from right apex.

LB $=$ Extrasystole from left base.

LA $=$ Extrasystole from left apex.

while the shift of the electrical axis was in the same direction as the shift in the anatomic axis in most types of beats when the heart's apex was moved from right to left of the long axis of the body with practically no rotation of the heart on its own long axis, the shift of the electrical axis 
was opposite in direction to that of the anatomic shift in most types of beats when this movement of the heart's apex was simultaneous with rotation of the heart on its own long axis (Table II). This result agreed with the observations of Boden and Neukirch (10), Meek and Wilson (11), and Nathanson (12) on the sinus beats. We have not only confirmed these results but have shown that they apply also to ectopic beats arising in various parts of the ventricle.

The variations in the shift of the electrical axes gave rise, as might have been expected, to reversal in the direction of the major deflection of the QRS group in a number of instances. The frequency of this occurrence has been summarized in Tables III and IV and typical examples are

TABLE III

On shifting heart's anatomic axis from right to left following reversals of direction of $Q R S$ noted

4 out of $7 \mathrm{~N}$ shifted - to + Lead I
4 out of $7 \mathrm{~N}$ shifted + to - Lead III
3 out of $6 \mathrm{RB}$ shifted + to - Lead III
1 out of $6 \mathrm{RA}$ shifted - to + Lead I
2 out of $6 \mathrm{RA}$ shifted + to - Lead III
1 out of 5 LA shifted + to - Leads I and II
1 out of $5 \mathrm{LB}$ shifted - to + Lead II
2 out of 5 LB shifted - to + Lead III

$\mathrm{N}=$ Normal complex.

$R B=$ Extrasystole from right base of heart.

R. $=$ Extrasystole from right apex of heart.

$L B=$ Extrasystole from left base of heart.

LA $=$ Extrasystole from left apex of heart.

TABLE IV

On shifting heart's anatomic axis from right to left and rotation on long axis from left ventricle anterior to right ventricle anterior following reversals of direction of $Q R S$ noted

$$
\begin{aligned}
& 1 \text { out of } 5 \mathrm{~N} \text { shifted }- \text { to }+ \text { Leads I and III } \\
& 1 \text { out of } 5 \mathrm{~N} \text { shifted }+ \text { to }- \text { Lead I } \\
& 2 \text { out of } 5 \mathrm{~N} \text { shifted }- \text { to }+ \text { Lead Il } \\
& 1 \text { out of } 4 \mathrm{RB} \text { shifted }- \text { to }+ \text { Lead I } \\
& 3 \text { out of } 4 \text { RB shifted }- \text { to }+ \text { Lead III } \\
& 1 \text { out of } 4 \text { RA shifted }- \text { to }+ \text { Lead I } \\
& 1 \text { out of } 4 \text { RA shifted }+ \text { to }- \text { Lead I } \\
& 3 \text { out of } 4 \text { RA shifted }- \text { to }+ \text { Lead II } \\
& 4 \text { out of } 4 \text { RA shifted }- \text { to }+ \text { Lead III } \\
& 1 \text { out of } 4 \text { LB shifted }- \text { to }+ \text { Leads I and II } \\
& 1 \text { out of } 4 \text { LB shifted }+ \text { to }- \text { Lead II } \\
& 2 \text { out of } 4 \text { LA shifted }- \text { to }+ \text { Lead I } \\
& 1 \text { out of } 4 \text { LA shifted }- \text { to }+ \text { Lead II }
\end{aligned}
$$

$\mathrm{N}=$ Normal complex.

$\mathrm{RB}=$ Extrasystole from right base of heart.

$\mathrm{RA}=$ Extrasystole from right apex of heart.

$\mathrm{LB}=$ Extrasystole from left base of heart.

$\mathrm{LA}=$ Extrasystole from left apex of heart. 
shown in Figures 6, 7 and 8, as well as in Figure 2 (cf. Lead III of RVA and LVA). Thus in Figure 6, when the heart's apex is shifted from the right to the left of the long axis of the body, the major initial deflection of the extrasystole increases in Lead I, decreases in Lead II and becomes inverted in Lead III; the QRS group of the sinus beat changes from a small inverted to an upright deflection in Lead I, decreases in size in Lead II, and becomes inverted in Lead III. In Figure 7 the major

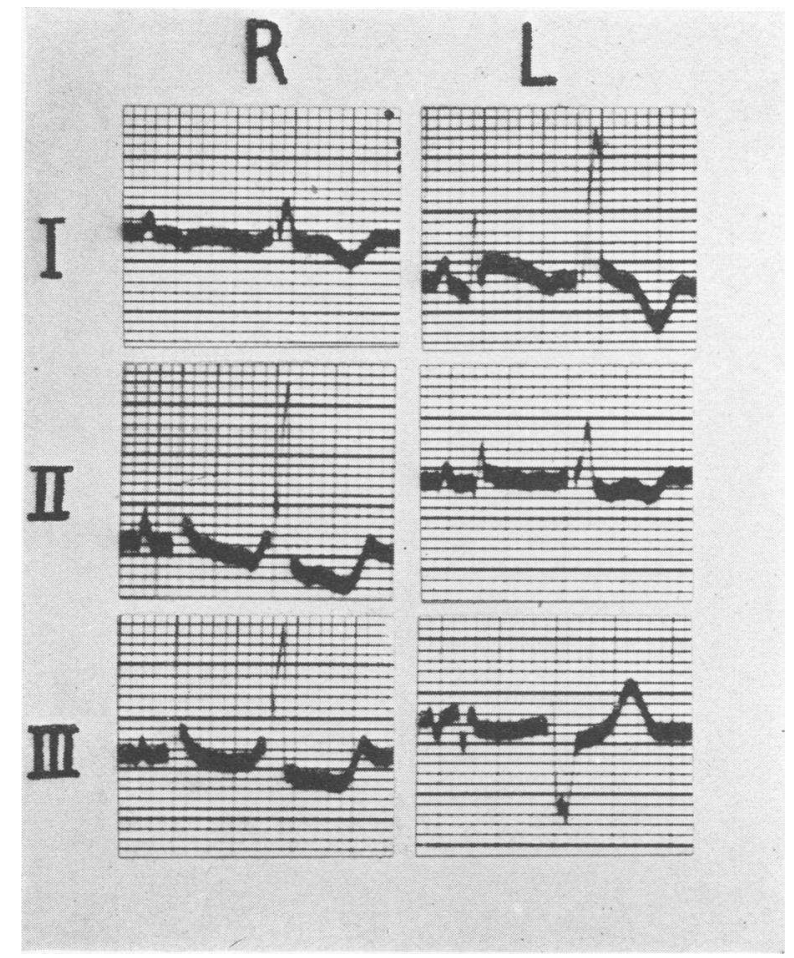

Fig. 6. The Electrocardiographic Appearance in the Ordinary Three Leads of the Sinus Beat and of the Extrasystole Induced in a Fixed Region of the Right Ventricular Apex in Two Positions of Heart (EXPERIMENT 1).

$\mathrm{R}$ and $\mathrm{L}$ have the same significance as in Figure 2. Note reversal of direction of QRS of the extrasystole in Lead III.

initial deflection of the extrasystole increases in size, especially in Lead II, while in Lead III it becomes inverted on shifting the heart's apex from the left to the right of the body's long axis. At the same time the sinus beat became more upright in all leads. In Figure 8 the changes are particularly striking since the major initial deflection is reversed in the two positions in Leads II and III, indicating a shift in the electrical axis of approximately $180^{\circ}$ in the plane of the leads. The sinus beat shows a similar shift in the direction of the QRS group. 


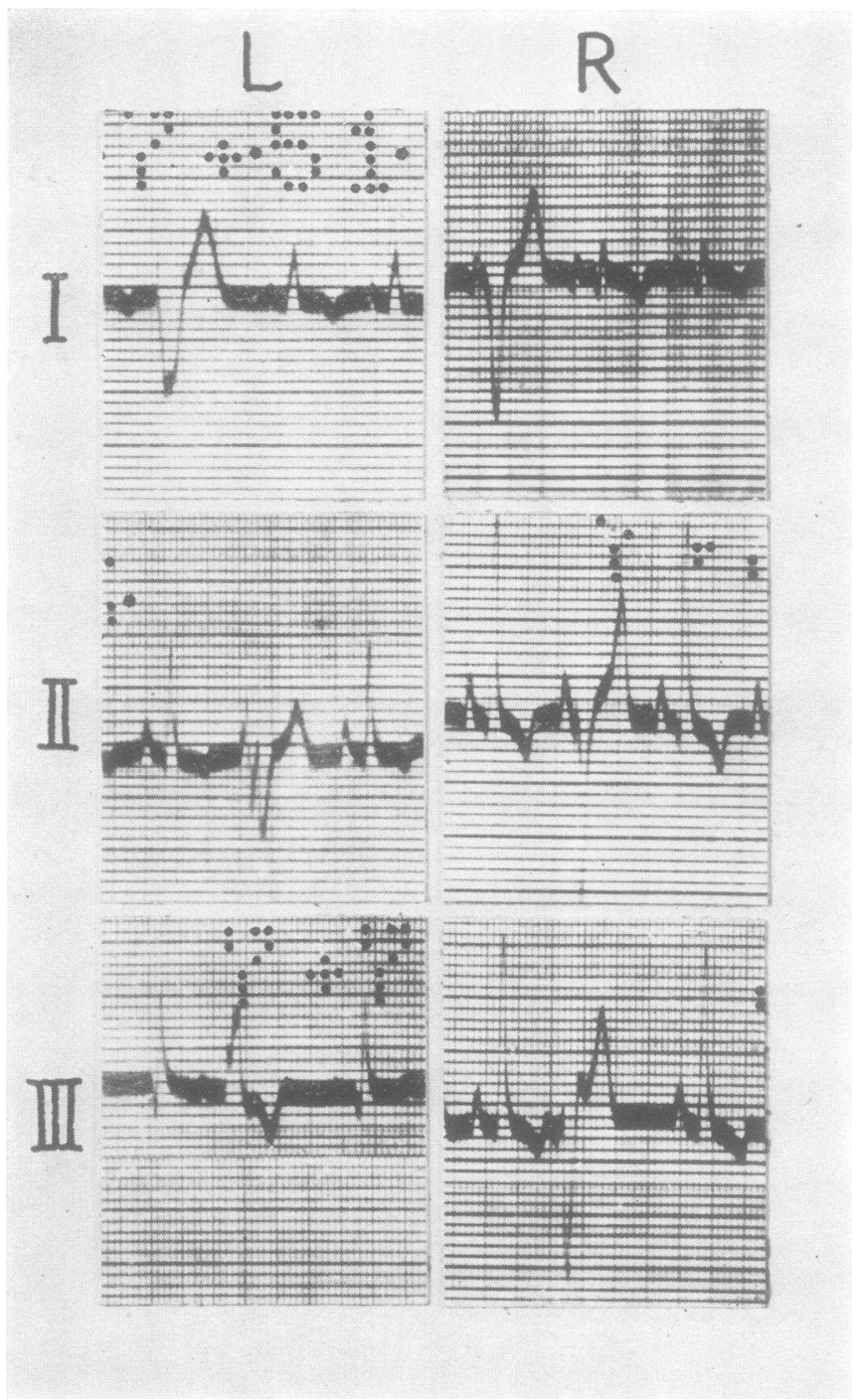

Fig. 7. The Appearance of the Sinus Beat and of the Extrasystole Induced in a Fixed Region of the Left Ventricular Base in Two PoSitions of Heart (EXPERIMENT 7).

$\mathrm{R}$ and $\mathrm{L}$ of same significance as in Fig. 2. Note reversal of QRS of the extrasystole in Lead III. 


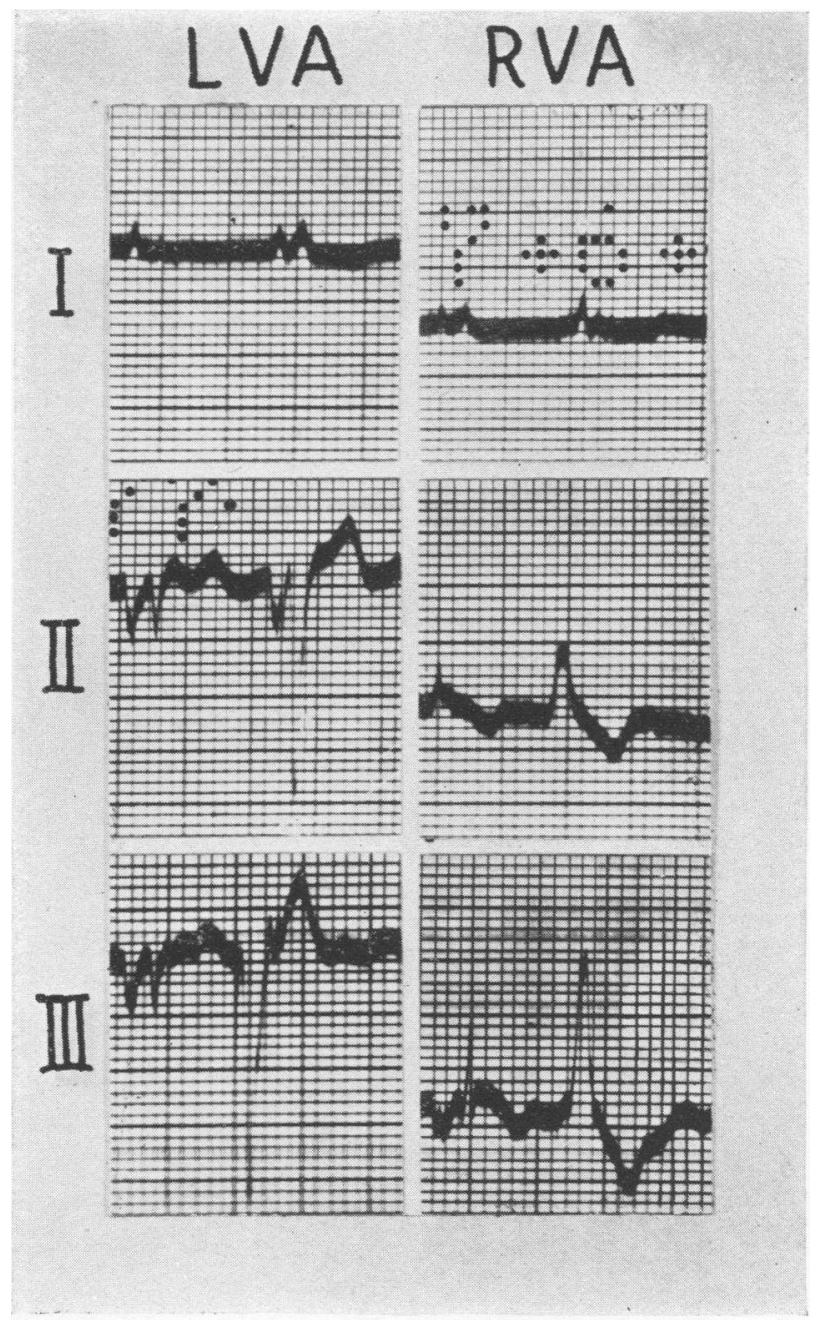

Fig. 8. The Electrocardiographic Appearance in the Ordinary Three Leads of the Sinus Beat and of the Extrasystole Induced in a Fixed Region of the Right Ventricular Apex (Experiment 6).

R.V.A. and L.V.A. of same significance as in Fig. 2. Note reversal of QRS in Leads II and III. 


\section{DISCUSSION}

The lack of proportionality of the changes, and the shift in opposite directions in the electrical axis of the various extrasystoles in a single experiment shows that the shift in the anatomic axis is not the only factor involved. The disparity arises from the fact that potentials set up by these extrasystoles act in three dimensions. Changes in the position of the heart therefore will change the projection of the vector in three dimensions cast on the plane formed by the three leads. ${ }^{3}$

The projection of this "three-dimensional" vector on the plane of the three leads varies monotonically with the inverse of the angle which it forms with this plane. When the position of the heart is changed, the angles between the three-dimensional vectors of the extrasystoles and the plane of the three leads are altered unequally and sometimes in such a way that the projections on this plane move in opposite directions, as can be seen from the diagram of Figure 9. This is particularly important

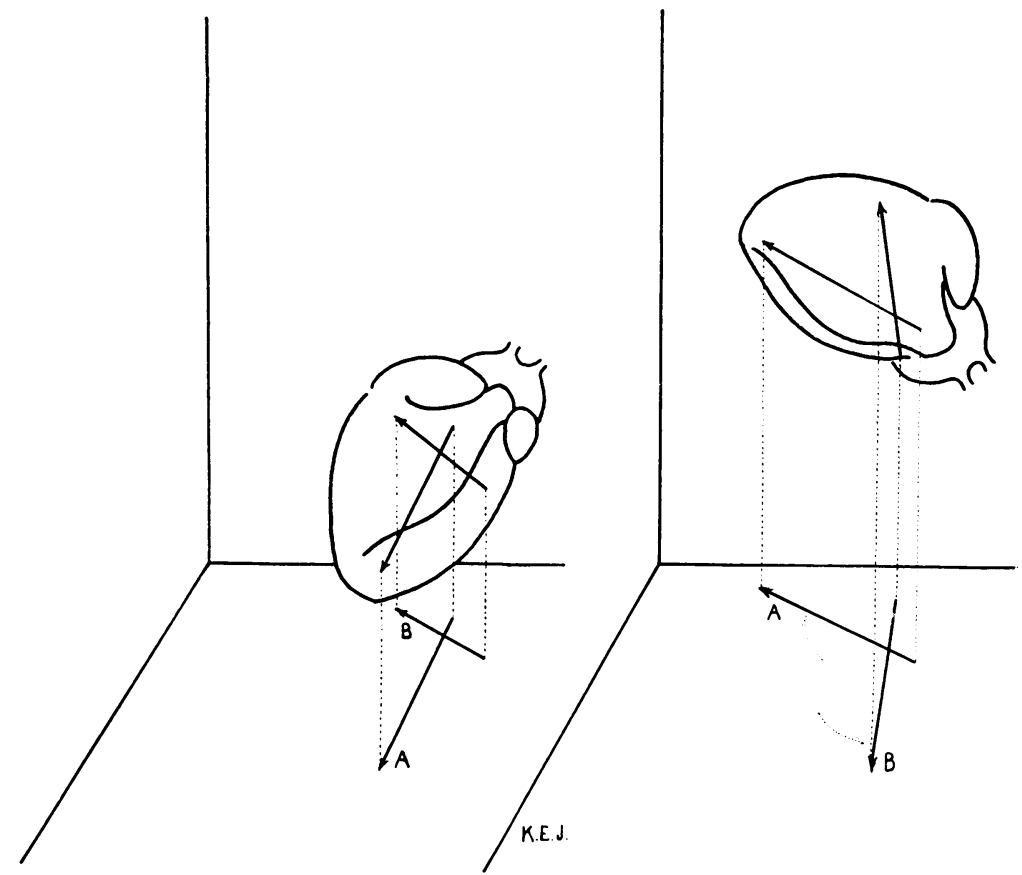

Fig. 9. Three-Dimensional Diagram Showing the Movement in Opposite Directions of the Projection on the Plane of Three Leads of the Three-Dimensional Vectors Produced by Two Extrasystoles When the Position of the Heart Was Changed.

$\mathrm{A}$ and $\mathrm{B}$ are the projections of the two vectors on the plane of leads.

${ }^{3}$ The projection on the plane of the three leads of the vector in three dimensions has been termed the electrical axis of the heart in this paper, since it is commonly so used. 
since rotation of the heart on its own long axis has such a tremendous influence, and since it is impossible to move the heart without changing the relation of the chambers to the plane of the leads.

In other words, we can view the movement of the heart in three dimensions as bringing into prominence the effect of new regions and decreasing the effect of the old ones. This may bring about a new balance in the plane of the leads in a direction opposite to the shift in the projection of the anatomic axis in this plane. Results such as presented in this report reemphasize the fact that the three standard leads constitute only two dimensions of the three dimensional potential differences set up by the heart.

The ability, by shifting the position of the heart, to reverse the direction of the major initial complex of ventricular extrasystoles evoked from fixed spots shows that the position of the heart and its configuration cannot be ignored in analyzing the origin of extrasystoles and the location of bundle branch block. Furthermore, these results would tend to show that the configuration of the electrocardiograms of the extrasystoles induced in the human heart by Barker, Macleod and Alexander (3) and by Marvin and Oughterson (4) may have been distorted by rotation and change in position of the heart or by dilatation of the ventricular chambers, and it would be hazardous to assume without further proof that their results can be applied to the normal human heart.

Although the changes observed in our experiments are probably more marked than occur in man they are nevertheless applicable since rotation of the heart on its own long axis has the greatest influence on the electrical axis. Hypertrophy and dilatation by changing the relation of the heart's chambers to each other probably have the same influence as rotation of the heart on its axis, and such changes are probably as marked as those produced by these rotations. In favor of this view is the fact that deviations just as marked as in our experiments sometimes occur in the electrical axis of the sinus beats in human hearts.

The results confirm the impression previously stated, that changes in the heart's position resulting from displacement or from dilatation and hypertrophy of the ventricles may be responsible in many instances for the discrepancy between the electrocardiographic and postmortem diagnosis of bundle branch block. The frequency of preponderant left ventricular hypertrophy can explain the inversion of the major initial deflection in Lead III. In other words, the bundle branch block increases the duration of the QRS group and the preponderant hypertrophy determines the direction of the major deflections.

In the present state of knowledge and with the variability in direction of the QRS group which these experiments show can be produced by changing the position of the heart, it would be preferable not to attempt to locate the site of origin of ventricular extrasystoles and, for the same 
reason, bundle branch block. Instead, the diagnosis should be given as follows: intraventricular block of the so-called bundle branch block type.

We have found a similiar result with experimentally produced bundle branch block (Ackerman and Katz (15)).

\section{SUMMARY}

1. Studies were made of the effect, in the open-chested anaesthetized $\mathrm{dog}$, of changing the heart's position on the electrocardiograms of induced extrasystoles. The shifts of the heart were $(a)$ in an anteroposterior direction on the transverse axis at its base, $(b)$ in a lateral direction on the anteroposterior axis at its base with practically no rotation on its long axis, and $(c)$ rotation on its long axis (which runs from the base to apex) with some movement in a lateral direction on the anteroposterior axis at its base. The extrasystoles were induced in four regions, viz., the base and apex of the right and left ventricles.

2. The electrical axis of the various extrasystoles did not move the same amount nor always in the same direction when the heart was shifted. The electrical axis moved in the same direction as the anatomic axis in most types of beats when practically no rotation on the heart's own long axis accompanied the change in the heart's position. The electrical axis moved in a direction opposite to the anatomic axis in most types of beats when the heart was rotated on its own long axis at the same time.

3. Many times the direction of the major initial deflection of the extrasystoles was reversed in one or more leads.

4. The results are attributed to the unequal movements of the socalled three-dimensional vectors of the various extrasystoles when the heart was shifted. This resulted in an unequal and at times opposite movement of the projections of these three-dimensional vectors on the plane of the three leads.

5. The reversal in direction of the QRS group of ventricular extrasystoles, which of ten accompanied changes in the heart's position, shows (a) that the configuration and position of the heart cannot be ignored in analyzing the site of ventricular extrasystoles and bundle branch block in man, and $(b)$ that the direction of the QRS group of extrasystoles induced in the two patients reported with pericarditis and pericardial fistula may not be applied to the non-diseased human heart without consideration of the other factors involved.

6. On the basis of these results it would be unjustified to attempt to localize the site of ventricular extrasystoles or of bundle branch block from the direction of the major initial complex in the three leads of their electrocardiograms. 


\section{BIBLIOGRAPHY}

1. Lewis, T., Phil. Trans. Roy. Soc., B. 1915, ccvii, 247. The Spread of the Excitatory Process in the Vertebrate Heart. III. The Dog's Ventricle. Phil. Trans. Roy. Soc., B, 1916, ccvii, 284. IV. The Human Ventricle.

2. Wilson, F. N., Macleod, A. G. and Barker, P. S., Am. Heart J., 1932, vii, 305. The Order of Ventricular Excitation in Human Bundle-Branch Block.

3. Barker, P. S., Macleod, A. G. and Alexander, J., Am. Heart J., 1930, v, 720. The Excitatory Process Observed in the Exposed Human Heart.

4. Marvin, H. M. and Oughterson, A. W., Am. Heart J., 1932, vii, 471. The Form of Premature Beats Resulting from Direct Stimulation of the Human Ventricles.

5. Fahr, G., Arch. Int. Med., 1920, xxv, 146. An Analysis of the Spread of the Excitation Wave in the Human Ventricle.

6. Taussig, H. B., Bull. Johns Hopkins Hosp., 1929, xlv, 40. A Case of Bundle Branch Block Confirmed by Pathological Study.

7. Oppenheimer, B. S. and Pardee, H. E. B., Proc. Soc. Exper. Biol. and Med., 1920, xvii, 177. The Site of the Cardiac Lesion in Two Instances of Intraventricular Heart Block.

8. Hill, I. G. W., Quart. J. Med., 1930, xxiv, 15. Bundle Branch Block. A Clinical and Histological Study.

9. Katz, L. N., J. Am. Med. Assoc., 1931, xcvii, 1364. Recent Advances in the Interpretation of the Electrocardiogram.

10. Boden, E. and Neukirch, P., Arch. f. d. ges. Physiol., 1918, clxxi, 146. Elektrokardiographische Studien am isolierten Säugetier- und Menschenherzen bei direkter und indirekter Ableitung.

11. Meek, W. J. and Wilson, A., Arch. Int. Med., 1925, xxxvi, 614. The Effect of Changes in Position of the Heart on the QRS Complex of the Electrocardiogram.

12. Nathanson, M. H., Proc. Soc. Exper. Biol. and Med., 1931, xxviii, 766. Electrocardiographic Study of Movements of the Heart with Changes in Posture.

13. Zeisler, E. B., Proc. Soc. Exper. Biol. and Med., 1930, xxviii, 12. A Critique of Einthoven's Law in Electrocardiography.

14. Mahain, I., Les Maladais Organiques du Faisceau de His Tawara., Masson \& Co., Paris, 1931.

15. Ackerman, W. and Katz, L. N., Reversal in Direction of the QRS of Experimental Right Bundle Branch Block with Change in the Heart's Position. Am. Heart J., (In Press). 